MSD prevention, which enables to integrate MSD prevention programs into safety management system in nursing homes. The essential aspects are the established MSD prevention programs have to be a fully integrated into general and safety management system in the organisation and the magnitude of complexity of MSD risk factors (including behavioural-cultural aspects of MSD), like most culturally-based things, must be recognised, acknowledged and be managed.

\section{RISK ASSESSMENT FOR BACK PAIN AND LUMBAR DEGENERATIVE DISEASE IN KOREAN FIREFIGHTERS}

${ }^{1}$ Hyung doo Kim*, 'Dong Hyun Kim, ${ }^{1}$ Yon Soo An, ${ }^{1}$ Kyoung Sook Jeong, ${ }^{1}$ Yeon-Soon Ahn, ${ }^{2}$ Jin-ha Yoon. 'Department of Occupational and Environmental Medicine, Dongguk University Ilsan Hospital, Goyang, Korea; ${ }^{2}$ The institute for Occupational Health, Yonsei University College of Medicine, Seoul, Korea

\subsection{6/oemed-2018-ICOHabstracts.760}

Introduction The work of firefighters inflicts a burden on the lumbar spine and may even provoke low back pain. Although ageing could cause degenerative changes in lumbar spine, there have been only few studies about lumbar degenerative changes in firefighters by age. Therefore, we have investigated the risk of lumbar degenerative changes in firefighters by age, while comparing with hospital office workers (HOWs) as our control group.

Methods We selected 490 professional firefighters using random sampling method by gender, age, and job. Then, we surveyed risk factors, job and clinical status, and conducted MRI and physical examinations, which was carried out by radiology specialists. Back pain was classified by NIOSH classification. The degenerative changes of lumbar spine were diagnosed as one of degenerative changes such as, intervertebral disc herniation or degeneration, central canal stenosis, and neural canal stenosis. The odds ratio of age-related lumbar degenerative changes in firefighters and control groups with $20 \mathrm{~s}$ years old (reference group) was evaluated using the logistic regression analysis.

Results The odds ratio of lumbar degenerative changes was significantly high only in $50 \mathrm{~s}$ among HOWs but in all age groups among firefighters. The central canal stenosis was significantly high prevalence in all age group, and disc herniation and degeneration were significantly high after $40 \mathrm{~s}$ among firefighters.

Conclusion The lumbar degenerative changes in firefighters occur at early age. It may be due to occupational factors of firefighter. In essence, further studies are needed to prevent these degenerative changes.

This research was supported by the Field-oriented Support of Fire Fighting Technology Research and Development Program funded by National Fire Agency ('MPSS-소방안전-2017-87').

\section{WORK-DISABLING MUSCULOSKELETAL PAIN IN CHILEAN COPPER MINERS: A CROSS-SECTIONAL STUDY}

\footnotetext{
1,2Méndez José*, ${ }^{3}$ Carvalho Denise, ${ }^{3}$ Radon Katja. ${ }^{1}$ Codelco Chile, División Andina, Los Andes, Chile; ${ }^{2}$ Sociedad Chilena de Medicina del Trabajo, Santiago, Chile; ${ }^{3}$ Center for International Health, Ludwig-Maximilians-Universität München, Munich, Germany
}

Introduction mining workers are known to be exposed to ergonomic and psychosocial risks, which are related to musculoskeletal disorders. The aim of this study was to determine the prevalence of work disabling low-back pain during the last year among Chilean copper miners, and its relation to ergonomic and psychosocial factors.

Methods a cross-sectional study was conducted during year 2014 in Los Andes, Chile, with 343 miners (response rate 99.7\%), classified according to tasks into operative $(n=253)$ and administrative workers $(n=79)$. The adapted survey of Working Conditions and Health in Latin America, Nordic questionnaire of musculoskeletal pain and ISTAS-21 questionnaire of psychosocial risk were applied. The main outcome was disabling neck and/or back pain in the last 12 months (pain that did not allow performing work normally). Descriptive, bivariate (chi ${ }^{2}$ test) and logistic regression (multivariate) analyses controlling for potential confounding factors were performed.

Results from all male workers considered, 94\% had complete secondary/higher education. Prevalence of disabling neck and/or back pain in the last year was significantly higher in operative than administrative workers $(14.9 \%$; $=0.01)$, and no difference was observed between workers from the main company and subcontractors. In bivariate analysis, a statistically significant association was found between the presence of pain and low/medium job security $(17.8 \% ; \mathrm{p}=0.04)$ and high strain jobs $(29.3 \% ; \mathrm{p}<0.001)$. No statistically significant association was found between high ergonomic risk and musculoskeletal pain. In logistic regression analysis, workers in high strain jobs (OR 3.13; 95\% CI: 1.20 to 8.18) were at increased odds of disabling back pain, compared to low strain jobs.

Discussion high strain jobs might be related to disabling back pain in Chilean miners. Psychosocial factors could have even a greater importance than ergonomic factors in the development of musculoskeletal pain in Chilean miners. Psychosocial risks surveillance could be as important as ergonomic workplace evaluation in the prevention of disabling musculoskeletal pain.

\section{DESCRIPTION OF A SPINAL GROUP PERFORMED BY A VEHICLE ASSEMBLER IN BRAZIL}

JMAP Cidale*, M Nusbaum*. Santa Casa de Misericórdia de São Paulo, São Paulo, Brazil

\subsection{6/oemed-2018-ICOHabstracts.762}

Introduction Low back pain is considered to be the second most frequent cause of morbidity and disability, leading only to headache. It affects $70 \%-80 \%$ of the adult population at some point in life, especially those who are working in the labour market. By affecting the economically active population, it interferes with family income and company productivity. This is because it brings suffering to patients and their families, and entails costs resulting from falling productivity, unpaid days, medical and legal expenses, insurance payments and disability benefits.

Methods Ergonomic evaluation by the Michigan Biomechanical Model and Analysis of the results obtained in the spinal group formed by a multiprofessional team, created by an automobile industry to treat their workers with spine pathologies in the last 2 years.

Result The ergonomic evaluation confirmed the presence of significant biomechanical overload for the lumbar spine and 
causal relationship with the activities evaluated. Of the 26 participants evaluated in the group, $53 \%$ had no limitations in the range of spinal movements at the time of examination, $73 \%$ reported improvement in quality of life, and only $15 \%$ said they had the same pain and limitation as when they started to do the accompaniment in the column program. When questioned, $80 \%$ of the participants reported that improvement in both physical examination and in quality of life was due to the treatment and follow-up done in the spinal group.

Conclusion The study found an improvement in the quality of life, a significant reduction in functional limitation and absenteeism in the participants of the group. These results are compatible with those obtained by the group in previous years and are also in agreement with those cited in the literature.

\section{DEVELOPMENT OF A PROGRAM FOR PATIENTS WITH FUNCTIONAL RESTRICTIONS IN A BRAZILIAN COSMETIC INDUSTRY}

${ }^{1}$ KS Arakaki*, ${ }^{2} \mathrm{M}$ Nusbaum*, ${ }^{2} \mathrm{~L}$ Vendramel ${ }^{*}$. ${ }^{1}$ Fundação Getúlio Vargas, São Paulo, Brazil; ${ }^{2}$ Santa Casa de Misericórdia de São Paulo, São Paulo, Brazil

\subsection{6/oemed-2018-ICOHabstracts.763}

Introduction The group of people with functional restrictions in industries needs special care and is a management challenge for any manager in occupational medicine. Being them just restricted for a limited period of time or restricted for a long period like rehabilitated for other functions by the social security institute, Readmitted (returned from medical leave longer than 15 days for diseases related to work) or Judicially reintegrated; A Brazilian cosmetic industry decided to improve the way they handle with this group of people. Trying to find the compatible areas for each of the necessary restrictions and evaluating the medical follow-up that was being performed Methods Ergonomic evaluation with job mapping by visiting workplaces with the ergonomist and the occupational physician. Definition of the list of most common restrictions and the development of a follow-up model for these patients. First the physician evaluated the patient and understood the current restrictions. Second determined the compatible area after visiting and understanding the function. And then created a Job mapping based on the Medical recommendations/restrictions. The Patients needed to be evaluated in 15, 30 or 45 days based on the occupational physician evaluation.

Result The program provided 467 medical attendance; 61 employees were accompanied by the occupational physicians; 35 employees were diagnosed with Osteomuscular disease; 17 $(27,8 \%)$ recovered from illness during a year;

Discussion This pilot program proved to be effective in monitoring and helping workers with restriction.

\section{WALKING, SITTING AND STANDING TIME MEASUREMENT SYSTEM WITH FOOT PLANER PRESSURE TO EVALUATE A RISK FACTOR OF LOW BACK PAIN}

\footnotetext{
${ }^{1} T$ Tsutsuiं, ${ }^{2} Y$ Tsutsui, ${ }^{1}$ I Inoue, ' $S$ Horie. 'Dep. Health Policy and Management, IIES, Univ. of Occupational and Environmental Health, Japan; ${ }^{2}$ Health Care Centre, Hitachi Metals Ltd., Japan
}

10.1136/oemed-2018-ICOHabstracts.764
Introduction Prolonged walking, sitting and standing were thought a risk factor of low back pain. We wanted to measure the prolonged walking, sitting and standing time among workers at the workplace to evaluate their low back pain. But it was difficult to measure these times. Foot planter pressure shifts according to the walking, sitting and standing modes. We handmade an insole typed foot planter pressure data logger and algorithm to presume the walking, sitting and standing time from the foot planter pressure data.

Methods The foot planter pressure data logger was composed by two resistive pressure sensors, a microcontroller, a micro SD card memory and a lithium ion battery. The foot planter pressure data downloaded to a personal computer and analysed to presume walking, sitting and standing time using visual basic software. We validated the presumption time of walking, sitting and standing from $10 \mathrm{~min}$ real activity time of running, walking, static sitting, dynamic sitting, static standing, dynamic standing, and taking off the shoe, respectively. This protocol did three times by one parson.

Results Concordance rate between the presumption time and the real activity time was running $83 \% \pm 12 \%$, walking $100 \%$ $\pm 0 \%$, static sitting $96 \% \pm 6 \%$, dynamic sitting $100 \% \pm 0 \%$, static standing $93 \% \pm 6 \%$, dynamic standing $73 \% \pm 38 \%$, and taking off the shoe $97 \% \pm 6 \%$, respectively. To join the running and walking time to the walking time, the static and dynamic sitting time to the sitting time, the static and dynamic standing time to the standing time, the concordance rate was up to $98.5 \% \pm 3 \%, 100.2 \% \pm 3 \%$, and $100.2 \% \pm 7 \%$, respectively.

Conclusion The insole typed foot planter pressure data logger had almost 98\% concordance rate between the presumption time and the real activity time of walking, sitting and standing. We could measure the walking, sitting and standing time of workers during their working time.

\section{INJURIES AND MUSCULOSKELETAL DISORDERS AMONG YOUNG WORKERS IN THE BRICK KILNS OF NEPAL}

'SV Joshi*, ${ }^{2}$ SK Joshi. 'Gyani Health Care Centre, Lalitpur, Nepal; '2Department of Community Medicine, Kathmandu Medical College, Kathmandu, Nepal

\subsection{6/oemed-2018-ICOHabstracts.765}

Introduction Brick manufacturing is a labour intensive informal industry using young workers as the major work force in Nepal. Young workers are required to use physical strength, carry heavy loads and remain in a squatted posture for longer periods doing repetitive tasks posing threats to musculoskeletal system. The objective of this study was to study prevalence of musculoskeletal disorders and injuries among young workers in the selected brick kilns in Nepal.

Methods This was a cross sectional study design involving young workers 17 years and below of age working in brick kilns in Bhaktapur and Sarlahi districts of Nepal. Study group included 101 young workers from Bhaktapur district and 97 from Sarlahi district, whereas, 64 nonworking children from Bhaktapur and 43 from Sarlahi districts were selected as the comparison group. Standardised Nordic questionnaires for the analysis of musculoskeletal symptoms and questions on injuries were administered.

Result Lack of adequate physical infrastructures, poor working conditions with nonexistent safety procedures have posed risk to physical, metal and overall well-being of children. The risks 